\title{
On the Comprehensive Reactive Power Compensation System of Large-scale Grid-connected Wind Power
}

\author{
Li Chengwei, Zhou Zhe, Liu Mingyuan \\ Fuxin Power Supply Company of National Grid Liaoning Electric Power Co., Ltd \\ henxue1997@163.com
}

Keywords: Fuxin area; wind power; grid connection; reactive power compensation

\begin{abstract}
With the constant expansion of Fuxin grid scale and the rapid development of wind farms, problems related with wind farms become increasingly prominent and voltage of wind farm is affected by changes in wind power active output and reactive output. This paper takes Fuxin area as example to analyze the existing problems in reactive voltage control in large-scale centralized grid-connected wind power, study the great impact of large-scale centralized grid-connected wind power and propose countermeasures and suggestions.
\end{abstract}

\section{Basic Situation of Fuxin Wind Power Base}

Fuxin wind farms are mainly based in the northwest area of Fuxin and eastern Zhangwu. By the end of 2014, there has been a wind power capacity of 1,693,300 kilowatts in operation, among which there are $6220 \mathrm{kV}$ substation, $566 \mathrm{kV}$ substation that delivers power to load centers like Jinzhou and Shenyang by $220 \mathrm{kV}$ tie line. According to Fuxin City Plan, there will be an installed capacity of 16,167,000 kilowatts by 2015, among which 8.117 million $\mathrm{kW}$ are thermal power (increasing 6.3 million kilowatts) and 8.05 million kilowatts are new energy (increasing 6.25 million kilowatts); power generation will be as much as 58.1 billion $\mathrm{kWh}$.

\section{Present situation of reactive power control system in Fuxin area}

In 2012, AVC system debugging has been done in Fuxin area and automatic control over reactive resources and regulating equipment has been achieved. It enhanced the overall stability of voltage in the system and at the same time reduces effectively damages caused by reactive power flow. The system helps realize closed-loop control over the reactive power adjustment device. Readjustment over the reactive power distribution in the grid enables the grid to work in a more secure and economic condition.

With the constant expansion of Fuxin grid scale and the rapid development of wind farms, problems related with wind farms become increasingly prominent and voltage of wind farm is affected by changes in wind power active output and reactive output. In order to maximize the production of electricity, the power source would generally make no adjustment or restrictions over the active power, but reactive power can be adjusted by various compensation measures to achieve control and improvement of wind farm voltage. In order to achieve reasonable distribution of reactive compensation in wind farms and achieve adaptation and coordination with the structure and characteristic of grid connection of wind farm. It is necessary to solve the following problems related to the grid in Fuxin area:

(1) Reactive devices like wind farm SVG in Fuxin area should not have great impact on the stability of system voltage; in addition, most wind farms are connected to the grid in the end of the system and there must be certain unreasonable problems in function mode[1], making the voltage problems more prominent.

(2) The adjusting tolerance interval of SVQC devices in Fuxin area is 5 minutes, which cannot meet the needs of voltage fluctuations; in addition, there are still many other doubtful aspects in reactive power compensation switching principles.

(3) The "sufficient power generation and little power consumption" feature of Fuxin area will bring certain difficulties in the reactive voltage adjustment in Fuxin area. Currently capacities of 
some parallel capacitors are too large in Fuxin grid, leading to the over bus compensation after being put into operation and too low bus voltage after being cut off; manual adjustment is not timely enough and voltage exceeding problems.

Therefore, it is especially important to accelerate construction and coordinating control over the reactive devices in substations and wind farms in Fuxin area.

\section{Solution Design of Reactive Power Compensation in Ground Wind farms}

\section{Reactive Power Compensation and Solution Design of Ground Wind farms}

In order to take full advantage of reactive power of each wind turbine group in wind farms, it is necessary to research on the automatic voltage control system in wind farms and fully exploits the reactive power support capability of each wind turbine group.

The integrated automatic voltage control system in wind farms is a control system that adjusts reactive power compensation equipment and the wind turbine group itself according to the adjustment orders and signals of the grid-connected wind farms to achieve optimal control over the whole wind farm. In order to reduce the adverse effects of wind farms on the system, it is necessary to design an integrated automatic voltage control system for the wind farm.

\section{Status of Reactive Power Operation in Regional Wind Farms}

Wind farm construction in China generally adopts unified planning and phased implementation method, that is to construct in several stages after wind farms being selected. Since turbines in the same stage should be the same type from the same manufacture, same control devices and measures are taken in the control of turbines. Currently, the power coefficient of each turbine is adopted as the control target. According to the requirement of "technical roles for connecting wind farm to power network" by the State Grid Corporation, wind farms should control the voltage of connection point of wind farms within the range of $-3 \% \sim+7 \%$ of rated voltage within their capacity. Under any operation methods, reactive power should be ensure with certain adjustment capacity and the power coefficient changes between the range of 0.98 (ahead) and 0.98 (lagging) when the wind farm is running under rated voltage. Reactive power adjustment capacity of a single wind farm in wind power bases of above one million kilowatts capacity should be within the range determined by the power coefficient between 0.97 (ahead) and 0.97 (lagging) when the wind farm is running under rated voltage.

The output voltage of wind turbines is generally $690 \mathrm{~V}$. There is a box-type transformer that can increase voltage of each generator into $35 \mathrm{kV}$ and connect it with the $35 \mathrm{kV}$ bus line by integrated line. There are reactive power compensation devices in $35 \mathrm{kV}$ bus line. There is a load adjustment transformer in the main transformer.

\section{Hardware for automatic voltage reactive power control in wind farms}

The control device of automatic voltage reactive power control in wind farms consists of a background, server and network switch, etc. the original substation monitoring system and wind turbine monitoring system should exchange and control information by servers and automatic voltage monitoring system in wind farms.

Automatic voltage monitoring system in wind farms can control SVG, SVC, capacitors and transformers tap changer.

AVC server should be capable of accepting but voltage adjustment orders in the high voltage side by master scheduler AVC station and upload AVC sub-station-related information to the scheduling AVC sub-station; do adjustments according to the reflection speed of reactive power devices; also be capable of accepting information from reactive power sources and sent the calculated adjustment orders to devices like SVG.

Software for automatic voltage control of reactive power in regional wind farms

Software for automatic voltage control of reactive power in wind farms includes software for data collection, processing, communication and diagnose. Functions of the software are mainly:

(1) Calculation

Calculate the target value of reactive power outputted by wind farms according to the target value of bus voltage; 
Choosing the adjustment group;

Figuring out the reactive power output or voltage of each turbine and dynamic reactive power compensation device and hints about the main transformer tap position according to the order and distribution strategy.

(2) Safety lock: control over AVC sub-station should take full account of the security of all equipment in each wind farm, alarming and locking AVC when the equipment doesn't work properly.

(3) Event Record: AVC system should be able to record events

(4) In order to ensure the accuracy of recorded events, AVC system monitoring center should be capable of setting watch according to GPS communication by packet through serial ports and on the spot.

(5) Control over AVC when being put into operation and being cut off. There is a handle or platen in the main cabinet of the system by which the operator put into operation or cut off the AVC.

(6) Control mode of the system

Exit: cutting off AVC sub-station;

Test state: control mode of the system is open-loop and remote orders are issued by manual operation;

Closed loop: control mode of the system under cycle operation is closed-loop and remote orders do not require manual intervention;

Distant: AVC sub-station can receive and run according to orders from the main AVC station.

On the spot: AVC sub-station system operate according to the set voltage curve locally.

(7) Human machine interface: it is capable of running, monitoring the status, adjustment management and setting values, etc.

(8) System Security: The system should have strong ability in anti-computer virus and anti-invasion so as to provide access for hardware firewall and other security facilities.

(9) Connecting mode between equipment: AVC sub-station of the wind farm should communicate with master scheduler AVC by monitoring system where the communication agreements are generally IEC60870-5-104:2000. AVC sub-station terminal is 104 statute forwarding station, booster station monitoring system mostly use 101 statute and AVC sub-station side is 101 statute acquisition station.

(10) Communicating with master scheduler AVC and other systems.

(11) The safety lock condition: AVC master station will be locked automatically when there is protection malfunction or automatic movements of other reactive power devices. Control of AVC sub-station should take full consideration of the security of all equipment in the wind farms, alarming and locking AVC control when the device does not work properly. When any of the following conditions occurs, AVC sub-station stops outputting automatically and alarms until it recovers to normal condition.

\section{Expected target of integrated automatic voltage control system in regional wind farms}

As a kind of power source, wind farms are necessary to participate in reactive adjustment and voltage control of the system. Currently, there are mainly three measures for reactive power voltage adjustment in wind farms:

(1) Manual or automatic adjustment of booster transformer taps;

(2) Automatic control of the reactive power compensation device in wind farms;

(3) Joint control of wind turbine group reactive power adjustment and wind farm reactive power compensation device.

Under normal conditions the master station collects real-time data of voltage and reactive power in the relevant taps of wind farms by automatic adjustment SCADA according to the optimal target; take comprehensive and optimal measures and make voltage of each taps be mutual constrictions to realize the total target of reactive voltage control in wind farms and then take the above 3 methods to do optimal reactive power adjustment by integrated control system of wind farms. 


\section{Conclusion}

Ground adjustment AVC system takes full charge of the reactive power devices in regional grid and wind farms by ground adjustment master scheduler AVC. The primary target is to ensure the stable operation of regional grid; the second is to ensure the voltage and power coefficient of regional grid qualified; the last is to optimize the distribution of regional reactive power so as to reduce grid losses effectively.

Ground adjustment AVC system should be connected with reactive devices and automatic system of regional wind farms by network and form a unified whole logically so as to achieve unified and harmonized control. AVC strategies should be more reasonable and scientific to meet the reactive power needs of grid of the its own level and lower level, fully rearrange the reactive power resources within the grid and avoid reactive power compensation conflicts between all levels of grid, make reactive power adjustment trend more practical and increase further the balance of reactive power and quality of voltage so as to reduce system losses and ensure the secure, stable, quality and economic operation of the grid.

\section{References}

[1] Guo Qinglai. On the closed-loop control of phased reactive power voltage in electricity system, Beijing: Tsinghua University, 2005.

[2] Yang Hua, Liang Haifeng, Li Gengyin. Voltage coordination and control measures of wind farms in doubly-fed induction generator, Automation of Power System, 2010, 35 (2): 112-126.

[3] Gjengedal, T / Large-scale wind farms as power plant.Wind Energy, 2005, 8: 361-373.

[4] Li Xianqi. Research on the Stability of System Voltage of Variable Speed Wind turbine and Grid-connected Wind Power Plant [D]. Wuhan: Huazhong University of Science and Technology, 2008.

[5] Xu Yetong. Research on the Stability of Large-scale Wind Power Integration and Design of Regulation Scheme [D]. Beijing: North China Electric Power University, 2011.

[6] Li Hui, Wang Hesheng, Zhao Bin. Simulation Study on the Different Equivalent Model of Wind Power Plant [M]. Solar Power Journal, 2011,32 (7): 1005-1012. 